\title{
Dark Matter in Numerical Simulations of Galaxy Clusters
}

\author{
Thomas Kronberger* \\ Inst. for Astroph., Innsbruck, Austria \\ E-mail: Thomas.Kronberger@uibk.ac.at
}

S. Schindler, E. van Kampen, S. Kimeswenger, W. Domainko, W. Kapferer, M. Mair Inst. for Astroph., Innsbruck, Austria

\begin{abstract}
We have developed numerical models in order to understand properties of clusters of galaxies, especially the metal enrichment of the intra-cluster gas (Domainko et al. 2004, [3]). Here we present the numerical treatment of the dark matter component (DM) and the galaxy formation process in these combined N-body and hydrodynamic simulations. We study galaxy formation by identifying dark matter haloes and assigning a galaxy to them in a semi-numerical way. Variations of different model parameters, like feedback or parameters related to the strength of star bursts, change the distribution of the baryonic matter in the dark matter halo, influencing galaxy properties and therefore also the enrichment of the intra-cluster medium (ICM).

Clusters of galaxies consist of many complex physical objects, which are all interacting with each other, so numerical simulations are probably the best route to a better theoretical understanding. One unresolved issue is the origin of the heavy elements in the ICM. Neither the efficiency nor the time dependences of any proposed enrichment process, such as ram-pressure stripping or galactic winds, are known in detail. We aim at investigating this problem using our simulation method.
\end{abstract}

Baryons in Dark Matter Halos

5-9 October 2004

Novigrad, Croatia

${ }^{*}$ Speaker. 
Table 1: The parameters of the cosmological model

\begin{tabular}{ccccc}
\hline$\Omega_{\Lambda}$ & $\Omega_{M}$ & $\Omega_{b}$ & $h_{0}$ & $\sigma_{8}$ \\
\hline 0.7 & 0.3 & 0.04 & 0.7 & 0.93 \\
\hline
\end{tabular}

\section{The Simulations}

For the cosmological evolution of the dark matter component we use an N-body tree code, which allows us to follow the non-linear evolution of the density distribution. The cosmology we have chosen is a standard $\Lambda \mathrm{CDM}$ model, whose basic parameters are given in table 1 . The initial conditions are set by using the method of constrained Gaussian random fields. In these N-body simulations galaxy haloes are identified continuously using a local density percolation technique. This approach enables us to follow the merger histories of dark matter haloes of present-day galaxies. Percolation, also known as 'friends-of-friends', links together particles with a pairwise distance which is less than a given cut-off length. We use a linking length which is modified by the local density to avoid overmerging, i.e. the problem that substructure is not identified in dense regions. Once a dark matter halo is found, it is checked whether it forms a virialised structure. If this is the case, it is assumed that a galaxy can form within that halo and the dark matter particles are replaced by a single softened particle. This replacement is performed in order to avoid a numerical disruption of low mass haloes. To this particle various galaxy properties are assigned in a semi numerical way [1], i.e. using scaling relations or empirical rules. These galaxy properties are then, together with the gravitational potential of the dark matter, passed to a shock-capturing gridbased piecewise parabolic method (PPM) hydrodynamic simulation and interaction mechanisms of the galaxies with the ICM such as ram-pressure stripping and galactic winds are modelled. The simulations are performed on a nested grid, in order to get high resolution at the cluster centre.

\section{Investigation of Galaxy Formation Parameters}

In order to understand the importance of the choice of the galaxy formation parameters on the final galaxy population and hence on the enrichment processes we tested different models. One example is presented here. For the disc scale length $r_{d}$, the approximation of Mo et. al (1998) [2] is applied. The ratio of the angular momentum of a galactic disc to that of its dark matter halo is one parameter of this model, which we have varied. In model 1 each forming galactic disc gets the same angular momentum as the dark matter halo but in model 2 it gets only half of it. Hence the discs get smaller and denser in the second case, have more star formation and therefore a higher metallicity. This result is shown in figure 1 . As a consequence of the smaller discs, ram-pressure stripping becomes less efficient. But, as the ejected gas is more metalrich than for larger discs, the effect on the enrichment efficiency is smaller than expected from just looking at the functional dependences of ram-pressure stripping. The mass-loss rate is shown in figure 2 as a function of time. Clearly, the larger discs of model 1 get stripped more efficiently at the beginning until their gas discs become equal in size to those in model two and the mass-loss rates become comparable. 

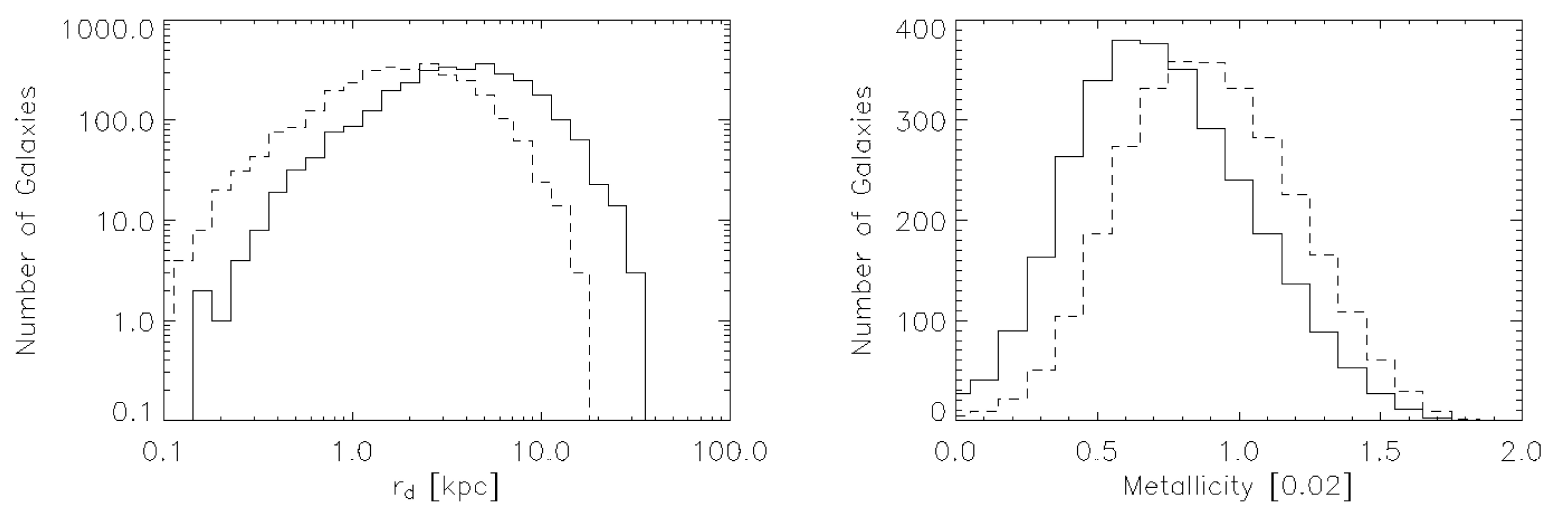

Figure 1: The distributions of the disc scale lengths (left) and of the metallicities due to disc star formation (right) are presented here for the two models mentioned above. The solid line is model 1, the dashed line model 2.

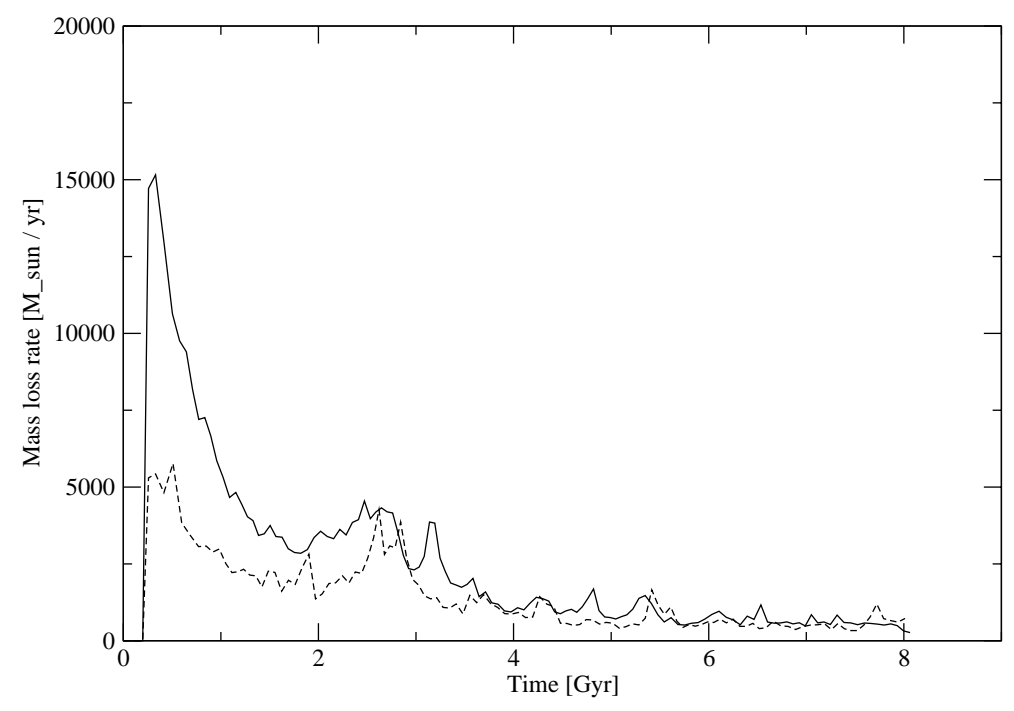

Figure 2: The mass-loss rate, in solar masses per year, due to ram-pressure stripping for the two models described in the text. Model 1 is the solid line, the dashed line is model 2. The first model shows a higher mass-loss rate at the beginning due to larger discs (see text).

\section{References}

[1] E. van Kampen, R. Jimenez, J.A. Peacock, 1999, MNRAS, 310, 43-56

[2] H.J. Mo, S. Mao, and S.D.M. White, 1998, MNRAS, 295, 319-336

[3] W. Domainko, W. Kapferer, S. Schindler, E. van Kampen, S. Kimeswenger, M. Ruffert, 2004, in Duc, Braine, Brinks, eds., IAU Symposium Series, Vol. 217 